\title{
Zur Frage der Abhängigkeit von Photostrom und Beleuchtungsstärke bei Photozellen und Photo-Sekundärelektronen-Vervielfachern
}

\author{
Von G. Kortüm und H. MaIeR ${ }^{1}$ \\ Aus dem Institut für Physikalische Chemie der Universität Tübingen \\ (Z. Naturforschg. 8a, 235-245 [1953]; eingegangen am 24. Februar 1953)
}

\begin{abstract}
Photozellen mit Sekundär-Elektronenvervielfachung (sog. Multiplier) wurden in den letzten Jahren zunehmend für die photometrische Messung kleiner Strahlungsintensitäten herangezogen. Bei den zu diesem Zweck entwickelten Methoden wird fast immer vorausgesetzt, daß Beleuchtungsstärke und vom Vervielfacher gelieferte Stromstärke einander innerhalb der verlangten Meßgenauigkeit von etwa $1 \%$ proportional sind. In Analogie zu früheren Messungen an Photozellen wird gezeigt, daß diese für den PrimärPhotoeffekt vorauszusetzende Proportionalität bei technischen Photozellen und Vervielfachern keineswegs immer vorhanden ist, sondern daß Abweichungen auftreten können, die diese Genauigkeit um mehr als eine Zehnerpotenz überschreiten. Photometrische Präzisionsmessungen sind mit Photozellen wie mit Vervielfachern nur möglich, wenn man echte Nullmethoden mit optischer Kompensation von Intensitätsänderungen benutzt.
\end{abstract}

S ekundärelektronenvervielfacher (SEV) wurden ursprünglich für die Fernsehtechnik entwickelt, werden aber heute auf Grund ihrer großen Empfindlichkeit überall da verwendet, wo nur kleine Lichtintensitäten oder kleine Intensitätsänderungen zur Verfügung stehen.

Das wesentlichste Anwendungsgebiet ist die Spektralphotometrie ${ }^{2}$. In der Literatur wird eine große Zahl von mit Vervielfachern ausgestatteten Photometern beschrieben. Teilweise wird eine normale Einzellenanordnung mit Ausschlagsmessung verwendet $^{3}$, teils eine Zweizellenanordnung mit Kompensationsschaltung ${ }^{4}$. Auch logarithmische Schaltungen zur direkten Extinktionsmessung ${ }^{5}$ (auf Grund der logarithmischen Strom-Spannungscharakteristik der Vervielfacher) wurden schon benutzt.

Besonders kleine Intensitäten stehen bei Streulichtmessungen ${ }^{6}$ und vor allem bei Raman- und Fluoreszenzspektren zur Verfügung, und daher werden vor allem hier Vervielfacher verwendet ${ }^{7-11}$.

\footnotetext{
${ }^{1}$ Auszug aus der Diplomarbeit von H. Maier, Tübingen 1953.

${ }^{2}$ R. W. Engstrom, J. opt. Soc. America 37, 420 [1947].

${ }^{3}$ B. Chance, Rev. Sci. Instruments 18, 601 [1947].

4 H. H. Großman u. E. W. Peterson, J. opt. Soc. America 39, 261 [1949].

5 M. H. Sweet, Elektronics 19, 105 [November 1946].

${ }^{6}$ B. A. Brice, M. Halwar u. R. Speiser, J. opt. Soc. America 40, 768 [1950].

7 C. H. Miller, D. A. Long, L. A. Woodward u. H. W. Thompson, Proc. phys. Soc. [London], Sect. A 62, 401 [1949].

${ }^{8}$ J. J. Heigl, J. F. Black u. B. F. Dudenbostol, Analytic. Chem. 21, 554 [1949]; 22, 154 [1950].
}

\section{Frühere Untersuchungen}

Die Frage der Abhängigkeit von Photostrom und Beleuchtungsstärke war im Laufe der Zeit Gegenstand vieler Untersuchungen, wenn sich auch der Gesichtspunkt, aus dem heraus die Fragestellung entstand, gewandelt hat. Zunächst war es nur eine wissenschaftliche Frage, die zur Klärung des physikalischen Mechanismus des Photoeffekts beitragen sollte, während später mit der Entwicklung der Anwendungsmöglichkeiten der praktische Gesichtspunkt den wissenschaftlichen überwog, und die Frage lautete: Inwieweit zeigt eine gegebene Zelle die für den primären Photoeffekt zu fordernde theoretische Proportionalität zwischen auffallender Beleuchtungsstärke und gemessenem Photostrom?

In den klassischen Arbeiten von Elster und Geitel $^{12}$, Lenard ${ }^{13}$, Griffith ${ }^{14}$, Richtmeyer ${ }^{15}$ und Ives ${ }^{16,17}$ sind die meisten verwendeten Methoden der Aufnahme der Photostrom-Beleuchtungs-

${ }^{9}$ R. H. Rank, M. A. Frenske, W. G. Braun, R. V. Wiegand u. D. Quiggle, Ind. Engng. Chem. 19, 700 [1947].

10 J. L. Lauer u. E. J. Rosenbaum, J. opt. Soc. America 41, 450 [1951].

${ }^{11}$ W. R. Busing, J. opt. Soc. America 42, 774 [1952]

12 J. Elster u. H. Geitel, Ann. Physik 48, 625 [1893].

${ }_{13}$ P. Lenard, Ann. Physik 8, 149 [1902].

14 J. O. Griffith, Philos. Mag. 14, 297 [1907].

15 F. K. Richtmeyer, Physic. Rev. 29, 71, 404 [1909].

${ }_{16}$ H. E. Ives, Astrophysic. J. 39, 428 [1914].

${ }^{17}$ H. E. Ives, S. Dushman u. E. Karrer, Astrophysic. J. 43, 9 [1916]. 
stärke-Kurven beschrieben, und eine große Anzahl der dabei auftretenden Schwierigkeiten aufgezeigt. Obwohl die Messungen dieser Autoren noch recht ungenau waren, fanden sowohl Griffith wie vor allem Ives bereits deutliche, außerhalb der Fehlergrenze liegende Abweichungen von der Proportionalität.

Diese Untersuchungen an Photozellen wurden in der Folgezeit von einer ganzen Reihe von Autoren bestätigt und ergänzt ${ }^{18-31}$. Die gemessenen Abweichungen schienen von der Belastung, dem Gasdruck, dem Elektrodenabstand, der Oberfläche der lichtempfindlichen Schicht und einer ganzen Reihe weiterer äußerer Faktoren, vor allem von Ladungsanhäufungen und dünnen isolierten Filmen photoelektrisch empfindlichen Materials auf den Zellwänden abhängig zu sein. Es wurden die verschiedenartigsten Abweichungen gefunden, ja, dieselbe Zelle konnte bei verschiedenen Belastungen oder Wellenlängen des zur Messung verwendeten Lichtes sowohl konvex wie auch konkav gekrümmt sein, so daß es nicht möglich war, eine allgemein gültige Beziehung zwischen Photostrom und Beleuchtungsstärke zu finden ${ }^{21,29}$.

Ebenso wie für die Photozellen ist natürlich auch für die Photoelektronenvervielfacher die Frage nach der Proportionalität zwischen Vervielfacherstrom und Beleuchtungsstärke von Bedeutung. Ein Vervielfacher ist ja nichts anderes als eine Vakuumzelle, deren Photostrom durch Sekundäremission multiplikativ erhöht wird. Daher ist von vornherein keine Proportionalität zu erwarten, wenn der von der Kathode emittierte Photostrom nicht der auffallenden Beleuchtungsstärke proportional ist. Aber selbst, wenn diese Voraussetzung erfüllt ist, kann die Linearität durch Nichtproportionalität der Sekundärvervielfachung, die z. B. durch Raumladungen in den letzten Vervielfacherstufen zustande kommen kann, gestört werden.

Bis jetzt ist unseres Wissens keine Arbeit bekannt

18 J. Kunz, Astrophysic. J. 45, 69 [1917]; Physic. Rev. 9, 175 [1917]; J. Kunz u. H. Stebbins, Physic. Rev. 7, 62 [1916].

${ }^{19}$ J. Elster u. H. Geitel, Physik. Z. 13, 468 [1912];

14, 471 [1913]; 15, 610 [1915].

${ }_{20}$ H. Rosenberg, Z. Physik 7, 18 [1921].

21 E. Steinke, Z. Physik 11, 215 [1922].

22 H. v. Halban u. L. Ebert, Z. Physik 14, 182 [1923].

${ }_{23}^{23}$ G. B. Welch, Physic. Rev. 32, 657 [1928].

24 G. H. Carruthers u. T. H. Harrison, Philos.

Mag. 7, 792 [1929]; 8, 210 [1929].

${ }_{25}$ W. S. Stiles, Philos. Mag. 7, 812 [1929].

${ }^{26}$ N. R. Campbell, Philos. Mag. 8, 63 [1929].

27 G. Kortüm, Physik. Z. 32, 417 [1931]. geworden, in der bei Vervielfachern Abweichungen von der Proportionalität angegeben wurden. Allerdings sollen schon mehrmals Vervielfacher mit gekrümmter Photostrom-Beleuchtungsstärke-Charakteristik beschrieben worden sein ${ }^{32}$.

Kluge, Beyer und Steyskal ${ }^{33}$ fanden einen Netzvervielfacher bei Bestrahlung mit weißem Licht proportional zwischen $10^{-8}$ und $10^{-3}$ Amp. Ausgangsstrom.

Auch Dieke und Crosswhite ${ }^{34}$ fanden bei den von ihnen untersuchten amerikanischen Vervielfachern keine Abweichungen.

Schließlich prüfte Engstrom ${ }^{2}$ eine Reihe von RCA-Vervielfachern auf Linearität. Er fand dabei zwischen $10^{-9}$ und $10^{-3} \mathrm{~A}$, maximale Abweichungen von $3 \%$, die aber seiner experimentellen Fehlerbreite entsprachen. Oberhalb von $10^{-3} \mathrm{~A}$ fand er ein sehr rasches Umbiegen zur Sättigung. Die obersten Werte wurden dadurch erhalten, daß das Licht nur kurzzeitig eingestrahlt wurde, um Ermüdung zu vermeiden.

Fast alle neueren Arbeiten über die Verwendung von Vervielfachern beziehen sich in der Frage der Linearität auf diese letzte Arbeit. Da jedoch von Photozellen bekannt ist, daß sie vielfach nicht linear arbeiten, ist dies auch von Vervielfachern zu erwarten. Wir haben deshalb die Proportionalität von modernen Photozellen und Vervielfachern zum Gegenstand einer sorgfältigen Untersuchung gemacht, da beabsichtigt ist, diese für die Messung kleiner Lichtströme bei Raman-Untersuchungen einzusetzen.

\section{Elektrische Apparatur}

\section{a) Spannungsstabilisierung}

Da die Sekundärelektronenausbeute eines Vervielfachers angenähert exponentiell mit der Stufenspannung ansteigt ${ }^{2}$ (mit Ausnahme der letzten

${ }^{28}$ H. v. Halban u. H. Geigel, Z. physik. Chem., Abt. B 96, 214 [1920].

${ }^{29}$ I. S. Preston u. L. H. McDermott, Proc. physic. Soc. [London], Sect. A 46, 256 [1934].

${ }^{30}$ G. A. B outry u. P. Gillod, Philos. Mag. 28, 163 [1939].

${ }^{31}$ I. S. Preston, Rev. Opt. théor. instrument 27, 513 [1948].

${ }^{32}$ Vgl. P. Görlich, Die Photozellen, Akademische Verlagsgesellschaft, Leipzig 1951, S. 194.

${ }_{33}$ W. Kluge, O. Beyer u. H. Steyskal, Z. techn. Physik 18, 219 [1937].

${ }^{34}$ G. H. Dieke u. H. M. Croßwhite, J. opt. Soc. America 35, 471 [1945]. 
Stufe zwischen der letzten Sekundärelektrode und der Anode), ist es notwendig, die Gesamtspannung äußerst konstant zu halten. Z. B. entspricht bei $100 \mathrm{~V} /$ Stufe einer Spannungsänderung von $1 \%$ eine Stromstärkenänderung von $4 \%$. In der Literatur sind deshalb zahlreiche Stabilisiergeräte für Vervielfacher beschrieben ${ }^{35}$.

In Abb. 1 ist das von uns gebaute Stabilisiergerät für Spannungen von $0-2100 \mathrm{~V}$ dargestellt. Es handelt sich um eine Kombination von Vakuumröhrenund Glimmstreckenstabilisierung. kondensator (6) $(2 \cdot 10 \mu \mathrm{F} \geqq 5 \mu \mathrm{F})$ geglättet. Durch den daran angeschlossenen Tiefpa $\beta(L=55$ Henry, $C=5 \mu \mathrm{F})(6 ; 7)$ wird die restliche Wechselkomponente ausgesiebt.

Das eigentliche Stabilisiergerät besteht aus zwei hochohmigen Endpentoden $(6 \mathrm{SJ} 7)(11 ; 12)$, deren Heizspannung $(6,3 \mathrm{~V})$ durch einen Eisenwasserstoffwiderstand stabilisiert wird. Die Gitterspannung der zweiten Röhre wird an einem Potentiometer abgegriffen (13). Steigt die Eingangsspannung, so steigt an der Röhre (12) auch der Innenwiderstand, und damit nimmt der Spannungsabfall an der Röhre zu. In Reihe mit den beiden Pentoden liegt die aus zwanzig

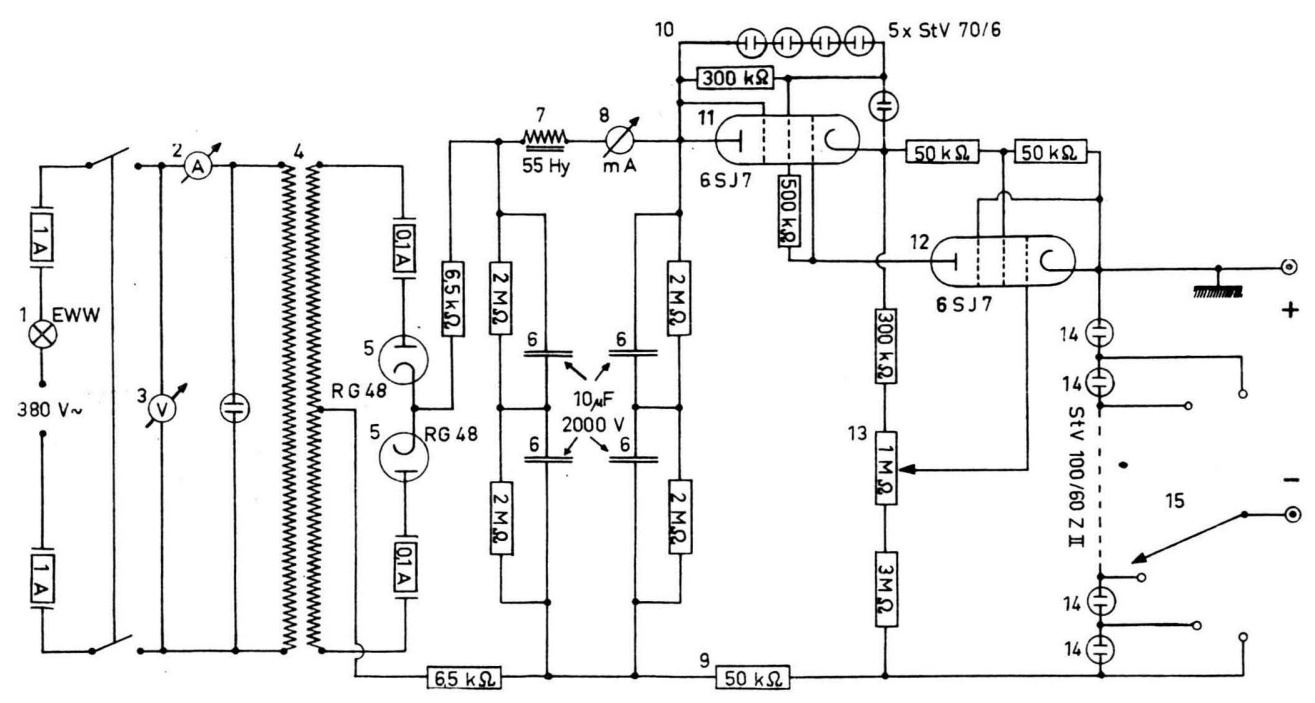

Abb. 1. Schaltskizze des Stabilisierungsgerätes für Spannungen von $0-2100 \mathrm{~V}$.

Die Netzspannung von $380 \mathrm{~V}$ wird mittels eines Eisenwasserstoffwiderstandes (1) auf $220 \mathrm{~V}$ am Eingang des Hochspannungstransformators (4) (220/7000 V) heruntergeregelt. Die Stromstärke im Transformator wird mit dem Amperemeter (2), die Spannung mit dem Voltmeter (3) kontrolliert. $380 \mathrm{~V}$ Primärspannung wurden gewählt, weil dadurch eine primärseitige Stabilisierung möglich ist, und erfahrungsgemä $B$ die Spannungsschwankungen zweier Phasen gegeneinander geringer sind, als die Schwankungen einer Phase gegen Erde. Die primärseitige Stromstabilisierung ist möglich, da die Änderungen im Stromverbrauch auf der Sekundärseite vernachlässigbar klein sind. Netzspannungsschwankungen von $5 \%$ werden dadurch auf Schwankungen von weniger als $1 \%$ reduziert. Die Sekundärspannung schwankt dadurch auch nur um maximal 1\%. Die Sekundärwechselspannung wird mittels zweier Hochspannungsgleichrichterröhren (5) (RG $48, U_{\mathrm{a} \max }=8 \mathrm{kV}, U_{\mathrm{h}}=5 \mathrm{~V}, I_{\mathrm{h}}=7 \mathrm{~A}$ ) in Doppelwegschaltung gleichgerichtet und durch den Lade-

${ }^{35}$ W. S. Plymale, jr. u. D. F. Hansen, Elektronics 23, 102 [Febr. 1950].
Glimmstrecken (14) StV 100/60 Z II bestehende eigentliche Endstabilisierung. An dieser Glimmstrecke kann die Gesamtspannung von 2100 Volt mit einem Stufenschalter (15) unterteilt und abgegriffen werden.

Die stabilisierende Wirkung einer Glimmstrecke beruht auf der bekannten Kennlinie einer selbständigen Entladung. Der Wechselstromwiderstand der verwendeten Strecken, der durch die Spannungsschwankung $\mathrm{d} U / \mathrm{d} i$ gegeben ist, liegt je nach der Frequenz der Schwankung zwischen 10 und $50 \Omega$. Der Vorwiderstand $R$ (9) muß so dimensioniert sein, daß etwa ein Drittel der Gesamtspannung an ihm vernichtet wird. Ändert sich der aus der Glimmstrecke entnommene Strom um $\mathrm{d} i$, so ist die Spannungsänderung an ihr

$$
\mathrm{d} U=w \cdot \mathrm{d} i(w=\text { Wechselstromwiderstand }) .
$$

Im Falle einer maximalen Änderung der Stromentnahme um $10^{-4}$ A würde bei der vorliegenden Strecke von 20 Glimmstabilisatoren eine maximale Spannungsänderung von $20 \cdot 50 \cdot 10^{-4}=10^{-1}$ auftreten. Diese Änderung fiele zwar nicht sehr stark ins Gewicht, aber, wie unsere Messungen zeigen, ist die tatsächliche Spannungsänderung wesentlich geringer. 
Schwankt die Speisespannung $U_{\text {ges }}$ um $\mathrm{d} U_{\text {ges, }}$, so tritt an der Glimmstrecke die Spannungsänderung

$$
\mathrm{d} U=\mathrm{d} U_{\text {ges }} \cdot \boldsymbol{w} / R(R=\text { Vorwiderstand })
$$

auf. Im vorliegenden Fall ist das Verhältnis $w / R=$ $1 / 50$.

Man nimmt vielfach an, daß die stabilisierende Wirkung von Elektronenröhren durch die spontanen Schwankungen in der nachfolgenden Glimmstrecke aufgehoben oder zumindest herabgesetzt werde. Das ist aber nach unseren Erfahrungen nicht der Fall. Meßbare spontane Schwankungen treten nur bei mit Barium aktivierten Glimmstrecken auf.

Die Konstanz der Ausgangsspannung wurde einmal durch Messung des Spannungsabfalles an einem Parallelwiderstand mit dem Kompensationsapparat und zum anderen durch Anlegen eines Teiles der Spannung an die Schneiden eines Edelmann-Elektrometers untersucht. Bei der Prüfung mit dem Kompensationsapparat ergab sich Spannungskonstanz innerhalb $10^{-30} \%(=1 / 50 \mathrm{~V})$ der Gesamtspannung bei Änderung der Eingangsspannung von 380 Volt um $\pm 20 \mathrm{~V}(=5 \%)$. Eine Meßreihe mit dem Elektrometer ist in Abb. 2 graphisch dargestellt.

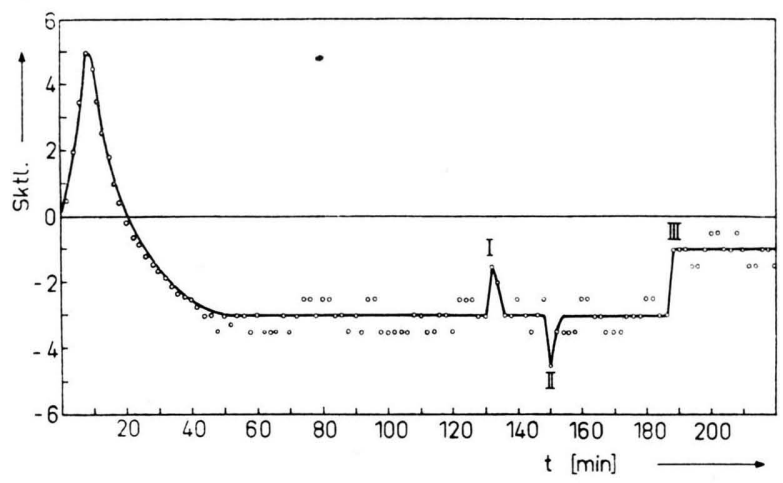

Abb. 2. Meßreihe zur Bestimmung der Konstanz der Ausgangsspannung mit Hilfe eines Edelmann-Elektrometers.
Als Abszisse ist die Zeit, als Ordinate die Stellung des Elektrometerfadens in Skalenteilen aufgetragen $\left(1\right.$ Sktl. $\left.=1 /{ }_{300} \mathrm{~V}\right)$. Die Schneidenspannung betrug $\pm 300 \mathrm{~V}$, der Elektrometerfaden lag auf Erdpotential. Nach einem Einlaufvorgang von etwa 30 Min. (Temperaturgang) ist die Spannung auf weniger als $1 / 300 \mathrm{~V}$ konstant. Bei I und II wurde die Netzspanung um + bzw. $-5 \%$ geändert. Bei III wurde ein zusätzlicher Verbraucher mit einem Stromverbrauch von $10^{-4}$ A parallel geschaltet. Die dargestellte Kurve stellt allerdings kein absolutes Maß für die Spannungskonstanz dar, da zum Nullpunkt symetrische Schwankungen nicht feststellbar sind.

b) Messung der Photoströme

\section{a) Photozellen}

Zur Messung der Photoströme wurde eine Kompensationsmethode benutzt, wie sie in Abb. 3 schematisch angegeben ist. Diese Methode ist von Empfindlichkeitsänderungen und Nullpunktsschwankungen anderer elektrometrischer Methoden unabhängig, da das Elektrometer lediglich als Nullinstrument dient; sie liefert deshalb sehr zuverlässige Resultate.

Das vom Spalt $\mathbf{S}$ eines Monochromators ausgehende Lichtbündel wird durch die Linse $\mathrm{L}$ parallelisiert und fällt auf die Zelle $\mathbf{Z}$. $\mathbf{W}_{1}$ ist ein Potentiometer, an dem die Zellspannung (aus dem Stabilisiergerät) abgegriffen werden kann. Sie wird durch ein statisches Voltmeter kontrolliert. $\mathrm{W}_{2}$ ist ein stabiler Hochohmwiderstand von $3 \cdot 10^{8} \Omega$. Es entsprechen daher $1 \mathrm{~V}$ Kompensationsspannung $3,33 \cdot 10^{-9} \mathrm{~A}$ Photostrom. $\mathrm{W}_{3}$ ist ein Präzisions-Kurbel-Dekaden-Widerstand von $11000 \Omega$ (einstellbar auf $0,1 \Omega$ ), der als Potentiometer zum Abgreifen der Kompensationsspannung dient. Letztere läßt sich zwischen 1,5 und $0,00002 \mathrm{~V}$, d. h. zwischen Photoströmen von $5 \cdot 10^{-9}$ und $6,7 \cdot 10^{-14}$ A einstellen und wird mit der Wolff-Brücke gemessen. Die beiden M $\Omega$-Widerstände dienen als Schutzwiderstände für das

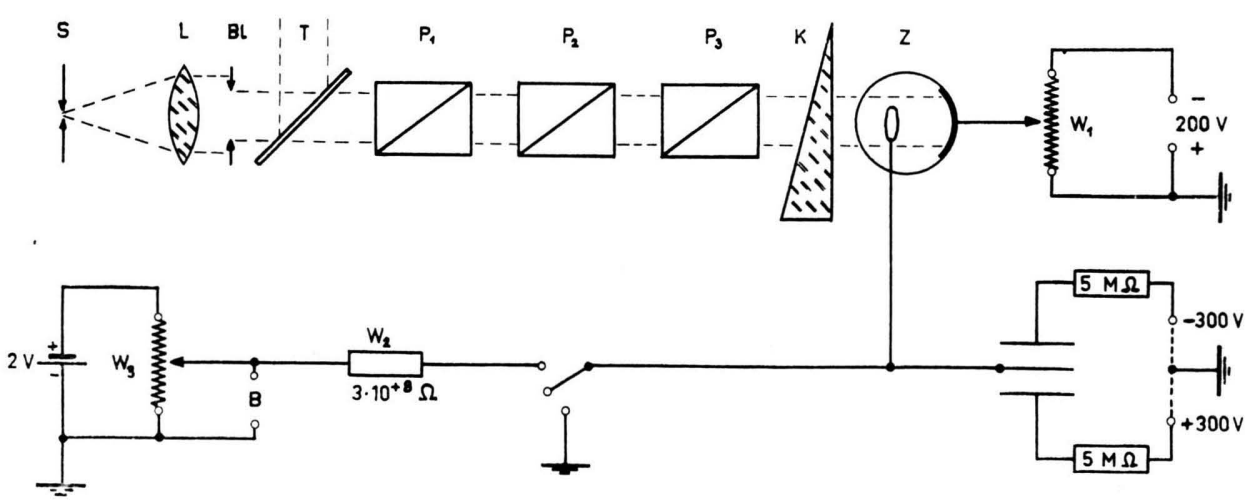

Abb. 3. Schematische Darstellung einer Kompensationsmethode zur Messung der Photoströme. 
Einfadenelektrometer nach Lutz-Edelmann, an dessen Schneiden je $300 \mathrm{~V}$ Hilfsspannung liegen. Die Genauigkeit ist u. a. begrenzt durch die Einstellgenauigkeit des Kompensationsapparates, die $0,00001 \mathrm{~V}$ beträgt. Zelle, Elektrometer, Kompensationswiderstand und die sie verbindenden Leitungsstücke sind zum Schutz gegen elektrostatische Störungen vollständig in ein geerdetes Metallgehäuse bzw. Metallrohre eingebaut.

\section{$\beta)$ Vervielfacher}

Der Ausgangsstrom eines Vervielfachers wird am einfachsten dadurch gemessen, daß man in den Anodenkreis ein empfindliches Galvanometer einbaut. Genauer aber ist die Messung eines Spannungsabfalles an einem Widerstand durch Kompensation.

Für unsere Messungen wurde ein Präzisionswiderstand von $10^{5} \Omega$ (umschaltbar auf $10^{4} \Omega$ ) in den Ausgang des Vervielfachers eingebaut, und der durch den Anodenstrom an ihm hervorgerufene Spannungsabfall mit der Wolff-Brücke gemessen. Die Spannungsmessung ist in ihrer Genauigkeit nur durch die Ablesegenauigkeit der Brücke und durch die Konstanz des Widerstands begrenzt. Der letztere Faktor ist zu vernachlässigen. Die Einstellgenauigkeit der Brücke beträgt $10^{-5} \mathrm{~V}$, mit einer Genauigkeit von $10^{-1}$. Es können also Stromstärken bis zu $10^{-10} \mathrm{~A}$ gemessen werden, die dann auf $10^{-11} \mathrm{~A}$ genau bestimmt sind. Die Ablesegenauigkeit des Galvanometers, das als Nullinstrument dient, betrug aber nur $10^{-10} \mathrm{~A}$, so da $\beta$ sämtliche Messungen an Vervielfachern nur auf $10^{-10} \mathrm{~A}$ genau sind.

\section{Optische Apparatur}

Um eine Photozelle oder einen Elektronenvervielfacher auf Proportionalität zwischen Photostrom und Beleuchtungsstärke zu prüfen, braucht man eine Anordnung, mit der meßbare Intensitätsänderungen über große Bereiche mit großer Genauigkeit durchgeführt werden können. In Abb. 3 ist auch der optische Teil der Apparatur dargestellt.

Das von einer Niederdruck-Quecksilberlampe kommende Licht wird in einem Monochromator zerlegt. Durch Ausprobieren wurde diejenige Neigung des Brennerrohres der Lampe zur Vertikalen ermittelt, die den Bogen am ruhigsten brennen ließ. Das vom Austrittsspalt $\mathbf{S}$ des Monochromators kommende Licht wird durch eine Quarzlinse L parallelisiert und mit einer Blende Bl ein Bündel von etwa $5 \mathrm{~mm}$ Durchmesser ausgesiebt. Dieses parallele Lichtbündel fällt auf eine um $45^{\circ}$ gegen die Lichtrichtung geneigte Quarzplatte, die etwa $10 \%$ des auffallenden Lichtes reflektiert, so daß zwei Lichtbündel von stets gleichem Intensitätsverhältnis entstehen. Das Hauptbündel fällt nun auf ein feststehendes Polarisations-(GlanThompson)-Prisma $P_{1}$. Das darauffolgende Prisma $P_{2}$ ist drehbar und mit einem auf 10 Sek. ablesbaren Teil- kreis versehen. Auf $P_{2}$ folgt nochmals ein feststehendes Polarisationsprisma $\mathrm{P}_{3}$, das genau parallel zum Prisma $\mathrm{P}_{1}$ steht. Diese Drei-Prismenanordnung wurde gewählt, da die Möglichkeit einer scheinbaren Nichtproportionalität auf Grund einer möglichen Abhängigkeit der photoelektrischen Empfindlichkeit von der Schwingungsrichtung des polarisierten Lichtes ausgeschaltet werden muß $\beta^{6}$. Das von Prisma $P_{3}$ kommende Licht geht nun durch einen Graukeil K zur Photozelle.

Die Teilerplatte $\mathrm{T}$ dient nur zur Ablenkung eines Teils des Lichtes auf eine Vergleichszelle für die Eichung der Apparatur. Der Graukeil $\mathrm{K}$ diente einmal zur Eichung und Prüfung der Prismenanordnung und bei der eigentlichen Messung zur Vergrößerung des zur Verfügung stehenden Intensitätsbereiches.

Da $P_{1}$ und $P_{3}$ parallel zueinander stehen, wird bei Parallelstellung von $P_{2}$ zu $P_{1}$ und $P_{3}$ das Licht vom Analysator vollkommen durchgelassen, bei gekreuzter Stellung vollkommen gelöscht. In den Zwischenstellungen ist die Durchlässigkeit gegeben durch

$$
I / I_{0}=\cos ^{4} x,
$$

wenn $x$ das Azimut der Prismen $\mathrm{P}_{1}$ und $\mathrm{P}_{2}$ bzw. $\mathrm{P}_{2}$ und $\mathrm{P}_{3}$ bedeutet. Die bei zwei verschiedenen Azimuten $x_{1}$ und $x_{2}$ durchgelassenen Intensitäten verhalten sich also wie

$$
I_{1} / I_{2}=\cos ^{4} x_{1} / \cos ^{4} x_{2} .
$$

Die Gültigkeit dieses $\cos ^{4} x$-Gesetzes ist aber an verschiedene Voraussetzungen gebunden. Fehlerquellen sind vor allem Licht eines zu großen Öffnungswinkels, nicht genügende Zentrierung des Systems, nichtparallele Prismenendflächen und vor allem Streulicht ${ }^{36}$. Zwar lassen sich alle diese Fehlerquellen nicht vollständig eliminieren, aber durch genaue Justierung ist eine weitgehende Beseitigung der Fehler möglich. Der Spalt des Monochromators wird genau in die Brennebene der Kollimatorlinse eingestellt und die Linse $L$ so justiert, daß ein möglichst paralleles Bündel entsteht. Die Polarisationsprismen werden am besten dadurch justiert, daß man das vom Ausgangsspalt des Monochromators kommende Licht durch Reflexion an der Vorderseite der Prismen wieder auf den Spalt abbildet.

Für die Prüfung der Prismen auf Gültigkeit des $\cos ^{4} x$-Gesetzes wurde eine Zweizellenanordnung ähnlich der von Ebert und Kortüm ${ }^{37}$ gewählt.

Die Stellung vollständiger Auslöschung des Analysators wurde visuell bestimmt, da bei sehr kleinen Lichtstärken das Auge empfindlicher ist als eine Photozelle. Der Analysator wurde dann auf einen bestimmten Anfangswert eingestellt, und die Photoströme gegeneinander kompensiert. Der Graukeil wurde nun jeweils von einem bestimmten Anfangswert bis zu einem bestimmten Endwert verschoben, und zwar wurde immer derselbe Anfangs- und Endwert gewählt, so daß irgendwelche Ungenauigkeiten oder Abweichungen in der Steigung des Keils nicht in die Messung eingehen. Dann wurde der Analysator gedreht, bis die

${ }^{36}$ W. H. St eel, J. opt. Soc. America 41, 223 [1951].

${ }^{37}$ L. Ebert u. G. Kortüm, Z. physik. Chem., Abt. B 13, 105 [1931]. 
Kompensation mit Hilfe des Elektrometers wieder hergestellt war. Es wurde immer von kleineren nach größeren Azimuten, d. h. von größeren zu kleineren Lichtstärken gedreht, um ein Nachhinken der Zellen zu vermeiden. Dieser Prozeß wurde bei allen möglichen Anfangsazimuten durchgeführt, und das jeweilige Verhältnis $I_{1} / I_{2}=\cos ^{4} x_{1} / \cos ^{4} x_{2}$ berechnet. Gehorcht die Prismenanordnung dem $\cos ^{4}$-Gesetz, so muß bei beliebigem Anfangsazimut immer das gleiche berechnete Intensitätsverhältnis auftreten. Um immer in etwa demselben Bereich der Charakteristik der Zellen $\mathrm{zu}$ arbeiten, wurde die auf die Meßzelle fallende Beleuchtungsstärke durch in den Lichtweg gebrachte Grauscheiben in engen Grenzen konstant gehalten, und die elektrische Kompensation der Zellen gegeneinander nur unwesentlich geändert. Die Keilverschiebung war durch eine Noniusablesung auf weniger als $0,02 \%$ genau einstellbar. Die Eichmessungen ergaben, daß das $\cos ^{4}$-Gesetz für jeden Quadranten nur innerhalb gewisser Bereiche auf weniger als $0,05 \%$ genau erfüllt ist. Für den ersten Quadranten beispielsweise war es zwischen $20^{\circ}$ und $60^{\circ}$ Azimut exakt gültig, bei kleineren und größeren Azimuten traten meßbare Abweichungen auf. Daher wurde bei den Messungen immer nur dieser Bereich verwendet.

\section{Fehlerabschätzung}

Der Gesamtfehler der Messung setzt sich zusammen aus dem Ablesefehler der Lichtschwächung, deren Eichfehler, den Schwankungen in der Speisespannung und damit im Anodenkreis der Röhre, den Schwankungen des Anodenstroms auf Grund der Inkonstanz der Lichtquelle sowie den inneren Schwankungen der Photozelle oder des Vervielfachers. Diese Einzelfehler sind aber mit Ausnahme der ersten beiden nicht theoretisch faßbar, und daher bestimmt man am besten den Gesamtfehler aus der Ablesestreuung der Einzelmessung.

Da die Prüfung auf Linearität so vorgenommen wurde, daß der Teilkreis des Analysators jeweils nach Einstellung eines bestimmten Azimuts zurückgedreht wurde, bis etwa wieder das Anfangsazimut erreicht war und die dazu notwendige Konstanz der Intensität mit dem Graukeil oder mit Grauplatten erreicht wurde, setzt sich der Gesamtfehler additiv aus den Einzelfehlern der einzelnen Abschnitte zusammen. Der oberste Intensitätswert des untersten Abschnittes sei mit einem Fehler von $x$ Prozent behaftet. Dann ist der Fehler, mit dem der unterste Intensitätswert des nächsten Abschnitts behaftet ist, genau so groß, und dazu kommt nochmals der Ablesefehler der Anordnung.

Bei den kleinsten vorliegenden Stromstärken liegt der Ablesefehler des Amperemeters in der Größenordnung von $0,3-0,5 \%$. Dieser äußert sich aber, wie alle anderen Fehlerquellen, in der Streuung der Ablesung des Teilkreises. Diese Ablesestreuung liegt hier in der Größenordnung von $1-2^{\prime}$, während sie bei höheren Werten rasch gegen $30^{\prime \prime}$ konvergiert. Für die Lichtschwächung gilt nun

$$
I / I_{0}=\cos ^{4} x / \cos ^{4} x_{0} .
$$

Daher ist

$$
\mathrm{d} I / I=-\left(4 \cos ^{3} x \sin x \mathrm{~d} x\right) / \cos ^{4} x=-4 \operatorname{tg} x \mathrm{~d} x .
$$

Da tg $x$ immer kleiner als 1,5 ist und $\mathrm{d} x$ kleiner als $30^{\prime \prime}$ bzw. 2' bei den kleinsten Intensitätswerten, wird $\mathrm{d} I / I$ kleiner als $4 \cdot 1,5 \cdot 1,5 \cdot 10^{-4}$ bzw. kleiner als $4 \cdot 1,5 \cdot 6 \cdot 10^{-4}$. Der Meßfehler der einzelnen Intensitätsmessung ist also im allgemeinen sehr klein und liegt in der Größenordnung von $0,1 \%$. Nur bei den kleinsten Intensitäten erreicht er eine Größe von $0,4-0,5 \%$.

Für die Meßergebnisse bei Photozellen gilt dasselbe wie für die bei Vervielfachern. Bei sehr kleinen Photoströmen wird aber der obige Fehler durch die begrenzte Empfindlichkeit des Elektrometers wesentlich vergrößert. Die größte verwendete Elektrometerempfindlichkeit betrug $1 / 1000 \mathrm{~V} /$ Sktl., die kleinste gemessene Kompensationsspannung $1 / 400 \mathrm{~V}$. Bei einer Ablesegenauigkeit von $1 / 10$ Sktl. ist also der kleinste gemessene Wert mit einem Fehler bis zu $4 \%$ behaftet. Dieser Fehler geht allerdings mit steigendem Photostrom sehr rasch zurück.

\section{Meßergebnisse}

Zur Untersuchung gelangten acht Photozellen und sechs Vervielfacher. Ihre wichtigsten Eigenschaften und Daten sind in Tab. 1 bzw. 2 zusammengestellt.

Der Dunkelstrom der AEG-Zellen war im allgemeinen sehr hoch, so daß die zur Verfügung stehenden Intensitäten nur noch für einen kleinen Teil der Charakteristik ausreichten. Der hohe Dunkelstrom kommt vermutlich durch Spuren niedergeschlagenen Caesiums auf den Isolatoren im Inneren der Zellen zustande.

Die Messungen wurden so durchgeführt, daß die Lichtschwächung mit Polarisationsprismen und Graukeil gleichzeitig vorgenommen wurde. Dadurch war es möglich, einmal mit den Prismen immer in dem Bereich zu arbeiten, in dem das $\cos ^{4} x$-Gesetz exakt gültig war, und doch bei einem großen Intensitätsbereich die hohe Ablesegenauigkeit des Prismenteilkreises auszunutzen. Der Analysator wurde also von einem bestimmten Anfangsazimut (z. B. 


\begin{tabular}{|c|c|c|}
\hline Nr. & Beschreibung und Herkunft & allgemeine Daten \\
\hline I & Vakuumzelle, Zeiß-Ikon MC 100 sV Nr. 3831 & $\begin{array}{l}\text { blauempfindlich, Empfindlichkeit } 16,4 \mu \mathrm{A} / \mathrm{Lm} \\
\text { Dunkelstrom: } 7,5 \cdot 10^{-12} \text { A bei } 100 \mathrm{~V}\end{array}$ \\
\hline II & Vakuumzelle, Zeiß-Ikon MC 100 sV Nr. 8880 & $\begin{array}{l}\text { blauempfindlich, Empfindlichkeit } 16,4 \mu \mathrm{A} / \mathrm{Lm} \\
\text { Dunkelstrom: } 7,5 \cdot 10^{-12} \text { A bei } 100 \mathrm{~V}\end{array}$ \\
\hline III & Vakuumzelle, Zeiß-Ikon MC 100 sV Nr. 8907 & $\begin{array}{l}\text { blauempfindlich, Empfindlichkeit } 14,2 \mu \mathrm{A} / \mathrm{Lm} \\
\text { Dunkelstrom: } 1 \cdot 10^{-11} \text { A bei } 100 \mathrm{~V}\end{array}$ \\
\hline IV & Vakuumzelle, Zeiß-Ikon MC 100 sV Nr. 8985 & $\begin{array}{l}\text { blauempfindlich, Empfindlichkeit } 17,2 \mu \mathrm{A} / \mathrm{Lm} \\
\text { Dunkelstrom: } 6 \cdot 10^{-12} \text { bei } 100 \mathrm{~V}\end{array}$ \\
\hline V & Vakuumzelle, AEG Fz 12 VS, Nr. 3896 & $\begin{array}{l}\text { rotempfindlich, Empfindlichkeit } 25 \mu \mathrm{A} / \mathrm{Lm} \\
\text { Dunkelstrom: } 2 \cdot 10^{-10} \mathrm{~A} \text { bei } 90 \mathrm{~V}\end{array}$ \\
\hline VI & Vakuumzelle, AEG Fr 11 VS, Nr. 4523 & $\begin{array}{l}\text { blauempfindlich, Empfindlichkeit } 55 \mu \mathrm{A} / \mathrm{Lm} \\
\text { Dunkelstrom: } 1 \cdot 10^{-10} \mathrm{~A} \text { bei } 90 \mathrm{~V}\end{array}$ \\
\hline VII & Gasgefüllte Zelle, AEG Fz 12 GS, Nr. 3683 & $\begin{array}{l}\text { rotempfindlich, Empfindlichkeit } 100 \mu \mathrm{A} / \mathrm{Lm} \\
\text { Dunkelstrom: } 1,6^{\cdot 10^{-9}} \mathrm{~A} \text { bei } 90 \mathrm{~V} \text {. Zündspannung } 60 \mathrm{~V}\end{array}$ \\
\hline VIII & Gasgefüllte Zelle, AEG Fz 11 GS, Nr. 3499 & $\begin{array}{l}\text { blauempfindlich, Empfindlichkeit } 105 \mu \mathrm{A} / \mathrm{Lm} \\
\text { Dunkelstrom: } 1 \cdot 10^{-12} \text { A bei } 90 \mathrm{~V} \text {. Zündspannung } 155 \mathrm{~V}\end{array}$ \\
\hline
\end{tabular}

Tab. 1. Photozellen.

\begin{tabular}{|c|l|c|c|c|c|c|}
\hline Nr. & \multicolumn{1}{|c|}{ Bezeichnung } & Stufenzahl & $\begin{array}{c}\text { Betriebs- } \\
\text { spannung } \\
(\text { Volt })\end{array}$ & Verstärkung & $\begin{array}{c}\text { Dunkel- } \\
\text { strom } \\
\text { (Ampere) }\end{array}$ & $\begin{array}{c}\text { Spektral- } \\
\text { bereich } \\
(U)\end{array}$ \\
\hline I & $\begin{array}{l}\text { Vervielfacher nach Dr, } \\
\text { S c h a e t t i (ETH Zürich) }\end{array}$ & 10 & 2100 & $10^{5}$ & $5 \cdot 10^{-8}$ & $4000-7000$ \\
II & $\begin{array}{l}\text { Vervielfacher nach Dr. } \\
\text { M a u r r VpA 11 tp 69c-d }\end{array}$ & 11 & 1850 & $10^{6}$ & $1,5 \cdot 10^{-8}$ & $3500-9000$ \\
III & RCA 931 A & 9 & 1100 & $10^{6}$ & $2,8 \cdot 10^{-9}$ & $3000-7000$ \\
IV & RCA 931 A & 9 & 1100 & $10^{6}$ & $4 \cdot 10^{-8}$ & $3000-7000$ \\
V & RCA 931 A & 9 & 1100 & $10^{6}$ & $5 \cdot 10^{-8}$ & $3000-7000$ \\
VI & RCA 931 A & 9 & 1100 & $10^{6}$ & $5 \cdot 10^{-8}$ & $3000-7000$ \\
\hline
\end{tabular}

Tab. 2. Vervielfacher.

$\left.20^{\circ}\right)$ successive über verschiedene Zwischenstellungen zu einem bestimmten Endazimut (z. B. $60^{\circ}$ ) gedreht, und dann die dadurch erreichte Lichtschwächung durch Graukeil und Grauscheiben kompensiert, wodurch der Analysator wieder auf eine Anfangsstellung von etwa $20^{\circ}$ zurückgedreht werden konnte. Es wurde bei zwei verschiedenen Quecksilberlinien und bei weißem Licht einer Punktlichtlampe gemessen.

\section{Photozellen}

In Tab. $3 \mathrm{a}-\mathrm{c}$ ist für ein Beispiel aufgeführt: Die Kompensationsspannung in Volt, der Photostrom in Ampere, das gemessene Verhältnis der Intensität zur Intensität beim tiefsten gemessenen Wert, das berechnete Intensitätsverhältnis (Sehne an die Kurve), die reziproke momentane Steigung der Photostrom-Beleuchtungsstärke-Funktion $\left[\left(I_{2}-I_{1}\right) /\left(i_{2}-i_{1}\right)\right]$ und die Abweichungen der Meßwerte von den berechneten Werten in Prozent. a) Weißes Licht,

b) Grüne Quecksilberlinie (5460 $\AA$ ),

c) Blaue Quecksilberlinie (4368 $\AA$ ).

Die folgenden graphischen Darstellungen (Abb. 4 und 5) zeigen einmal die Abhängigkeit des Photostroms von der Beleuchtungsstärke und dann die reziproke Steigung der Photostrom-Beleuchtungsstärke-Charakteristik $\mathrm{d} I / \mathrm{d} i$.

Die relativ großen Abweichungen von der Proportionalität sind auch in der doppelt-logarithmischen Darstellung noch bemerkbar, kommen aber in den Kurven der Abb. 5 besonders deutlich zum Ausdruck.

In analoger Weise wurden die übrigen Photozellen untersucht. Von den sechs Vakuumzellen zeigte nur die in Tab. 3 angegebene starke Abweichungen von der Proportionalität, bei den übrigen waren die $\mathrm{Ab}$ weichungen geringer und überschritten selten die Grenze von $1-2 \%$ in einem Intensitätsbereich von 
a) weißes Licht

\begin{tabular}{|c|c|c|c|c|c|}
\hline $\begin{array}{l}\text { Kompen- } \\
\text { sations- } \\
\text { spannung } \\
\text { (Volt) }\end{array}$ & $\begin{array}{c}\text { Photostrom } \\
\text { (Ampere) }\end{array}$ & $I_{\text {gem. }}$ & $I_{\text {her. }}$ & $\frac{I_{2}-I_{1}}{i_{2}-i_{1}}$ & $\begin{array}{c}I_{\text {gem. }}-I_{\text {ber. }} \\
I_{\text {gem. }} \\
\cdot 100\end{array}$ \\
\hline 0,900 & $3,00 \cdot 10^{-9}$ & 31,94 & 31,94 & 31,5 & - \\
\hline 0,800 & 2,6 & 28,79 & 28,38 & 32,0 & $+1,3$ \\
\hline 0,700 & 2,3 & 25,59 & 24,83 & 33,4 & 3,0 \\
\hline 0,600 & 2,0 & 22,25 & 21,27 & 35,3 & 4,5 \\
\hline 0,500 & 1,6 & 18,72 & 17,71 & 36,0 & 5,2 \\
\hline 0,400 & 1,3 & 15,12 & 14,15 & 36,6 & 6,5 \\
\hline 0,350 & 1,1 & 13,29 & 12,38 & 36,8 & 7,0 \\
\hline 0,300 & $1,00 \cdot 10^{-9}$ & 11,45 & 10,60 & 37,0 & 8,0 \\
\hline 0,250 & $8,3 \cdot 10^{-10}$ & 9,60 & 8,83 & 37,6 & 8,0 \\
\hline 0,200 & 6,7 & 7,72 & 7,05 & 38,0 & 8,8 \\
\hline 0,180 & 6,0 & 6,96 & 6,34 & 38,0 & 9,0 \\
\hline 0,160 & 5,4 & 6,20 & 5,62 & 38,5 & 9,6 \\
\hline 0,140 & 4,7 & 5,43 & 4,91 & 39,0 & 9,5 \\
\hline 0,120 & 4,0 & 4,65 & 4,19 & 38,5 & 10,0 \\
\hline 0,100 & 3,3 & 3,88 & 3,48 & 39,0 & 10,0 \\
\hline 0,080 & 2,7 & 3,10 & 2,77 & 40,0 & 11,0 \\
\hline 0,060 & 2,0 & 2,30 & 2,05 & 39,0 & 11,0 \\
\hline 0,050 & 1,6 & 1,91 & 1,69 & 38,0 & 12,0 \\
\hline 0,040 & 1,3 & 1,53 & 1,34 & 39,0 & 13,0 \\
\hline 0,030 & $1,00 \cdot 10^{-10}$ & 1,14 & 0,99 & 41,0 & 14,0 \\
\hline 0,020 & $6,7 \cdot 10^{-11}$ & 0,73 & 0,63 & 40,0 & 14,0 \\
\hline 0,015 & 5,0 & 0,53 & 0,45 & 42,0 & 15,0 \\
\hline 0,010 & 3,3 & 0,32 & 0,28 & 44,0 & 12,0 \\
\hline 0,0075 & 2,5 & 0,21 & 0,19 & 44,0 & 10,0 \\
\hline 0,005 & 1,6 & 0,10 & 0,10 & - & - \\
\hline
\end{tabular}

b) Grüne Quecksilberlinie (5460 ̊)

\begin{tabular}{|c|c|c|c|c|c|}
\hline $\begin{array}{c}U_{\mathrm{k}} \\
(\text { Volt })\end{array}$ & $\begin{array}{c}i_{\mathrm{Ph}} \\
\text { (Ampere) }\end{array}$ & $I_{\text {gen. }}$ & Iher. & $\frac{I_{2}-I_{1}}{i_{2}-i_{1}}$ & $\begin{array}{c}\frac{I_{\text {gem. }}-I_{\text {ber. }}}{I_{\text {gem. }}} \\
\cdot 100\end{array}$ \\
\hline 0,200 & $6,7 \cdot 10^{-10}$ & 9,16 & 9,16 & 44,5 & - \\
\hline 0,180 & 6,0 & 8,27 & 8,22 & 44,0 & $+\quad 0,6$ \\
\hline 0,160 & 5,4 & 7,39 & 7,29 & 45,0 & $+1,3$ \\
\hline 0,140 & 4,7 & 6,49 & 6,36 & 44,5 & $\begin{array}{r}+\quad 2,0 \\
+\end{array}$ \\
\hline 0,120 & 4,0 & 5,60 & 5,44 & 45,0 & $\begin{array}{r}2,7 \\
+\quad 2\end{array}$ \\
\hline 0,100 & 3,3 & 4,70 & 4,52 & 45,5 & $+\quad 3,9$ \\
\hline 0,080 & 2,7 & 3,79 & 3,60 & 45,5 & $+5,0$ \\
\hline 0,060 & 2,0 & 2,88 & 2,68 & 46,0 & $+7,0$ \\
\hline 0,050 & 1,6 & 2,42 & 2,22 & 47,0 & $+8,2$ \\
\hline 0,040 & 1,3 & 1,95 & 1,76 & 48 & $+10,0$ \\
\hline 0,030 & $1,0 \cdot 10^{-10}$ & 1,47 & 1,30 & 50 & $+11,5$ \\
\hline 0,020 & $6,7 \cdot 10^{-11}$ & 0,97 & 0,84 & 52 & $+13,5$ \\
\hline 0,015 & 5,0 & 0,71 & 0,61 & 54 & $+14,2$ \\
\hline 0,010 & 3,3 & 0,44 & 0,38 & 56 & $+13,6$ \\
\hline 0,0075 & 2,5 & 0,30 & 0,27 & 60 & $+10,0$ \\
\hline 0,005 & 1,6 & 0,15 & 0,15 & - & - \\
\hline
\end{tabular}

c) Blaue Quecksilberlinie ( $4358 \AA$ )

\begin{tabular}{|c|l|r|r|r|c|}
\hline $\begin{array}{c}U_{\mathrm{k}} \\
(\text { Volt })\end{array}$ & $\begin{array}{c}i_{\mathrm{Ph}} \\
\text { (Ampere) }\end{array}$ & $I_{\text {gem. }}$ & $I_{\text {ber. }}$ & $\mathrm{d} I / \mathrm{d} i$ & $\frac{I_{\text {gem. }}-I_{\text {ber. }}}{I_{\text {gem. }}}$ \\
& & & & & -100 \\
\hline & & & & \\
0,320 & $1,06 \cdot 10^{-9}$ & 11,55 & 11,55 & 34,5 & - \\
0,300 & $1,00 \cdot 10^{-9}$ & 10,86 & 10,82 & 34,0 & $+0,4$ \\
0,280 & $9,3 \cdot 10^{-10}$ & 10,18 & 10,10 & 34,0 & $+0,5$ \\
0,260 & 8,7 & 9,50 & 9,37 & 34,5 & $+1,5$ \\
0,240 & 8,0 & 8,81 & 8,64 & 35,5 & $+1,9$ \\
0,220 & 7,3 & 8,10 & 7,91 & 34,5 & $+2,5$ \\
0,200 & 6,7 & 7,41 & 7,18 & 36,0 & $+3,0$ \\
0,180 & 6,0 & 6,69 & 6,45 & 36,0 & $+3,5$ \\
0,160 & 5,4 & 5,97 & 5,74 & 36,5 & $+3,8$ \\
0,140 & 3,7 & 5,24 & 5,02 & 37,0 & $+4,5$ \\
0,120 & $\mathbf{4} 0$ & 4,50 & 4,25 & 37,0 & $+5,5$ \\
0,100 & 3,3 & 3,76 & 3,53 & 38,0 & $+6,0$ \\
0,080 & 2,7 & 3,00 & 2,81 & 38,5 & $+6,3$ \\
0,060 & 2,0 & 2,23 & 2,09 & 39,0 & $+6,4$ \\
0,050 & 1,6 & 1,84 & 1,73 & 38 & $+6,0$ \\
0,040 & 1,3 & 1,46 & 1,37 & 38 & $+6,4$ \\
0,030 & $1,0 \cdot 10^{-10}$ & 1,08 & 1,01 & 39 & $+6,6$ \\
0,020 & $6,7 \cdot 10^{-11}$ & 0,69 & 0,64 & 40 & $+7,0$ \\
0,015 & 5,0 & 0,49 & 0,46 & 38 & $+6,0$ \\
0,010 & 3,3 & 0,30 & 0,28 & 40 & $+6,5$ \\
0,0075 & 2,5 & 0,20 & 0,19 & 40 & $+5,0$ \\
0,005 & 1,6 & 0,10 & 0,10 & - & - \\
& & & & & \\
\hline
\end{tabular}

Tab. 3. I Vakuumzelle Zeiss-Ikon MC $100 \mathrm{sV}$ Nr. 3831, Betriebsspannung $100 \mathrm{~V}$, Dunkelstrom $7,5 \cdot 10^{-12}$.
2-3 Zehnerpotenzen. Von den beiden untersuchten gasgefüllten Zellen zeigte die eine (AEG Fz 12 GS) Abweichungen bis zu 4\%, während die andere innerhalb der Fehlergrenze der Methode linear war.

\section{Vervielfacher}

Die Bezeichnungen in Tab. $4 \mathrm{a}-\mathrm{c}$ sind dieselben wie bei den Photozellen.

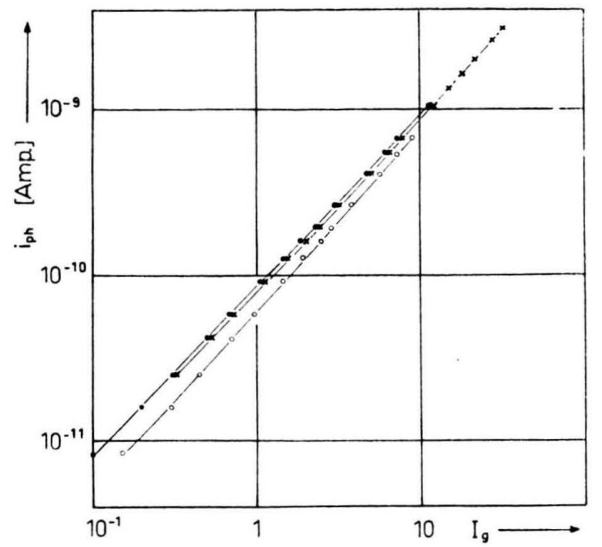

Abb. 4. Abhängigkeit des Photostromes von der Beleuchtungsstärke. MC 100 sV Nr. 3831; $\bigcirc \mathrm{Hg} 5460$ A, - $\mathrm{Hg} 4338 \mathrm{~A}$, $\times$ weißes Licht.

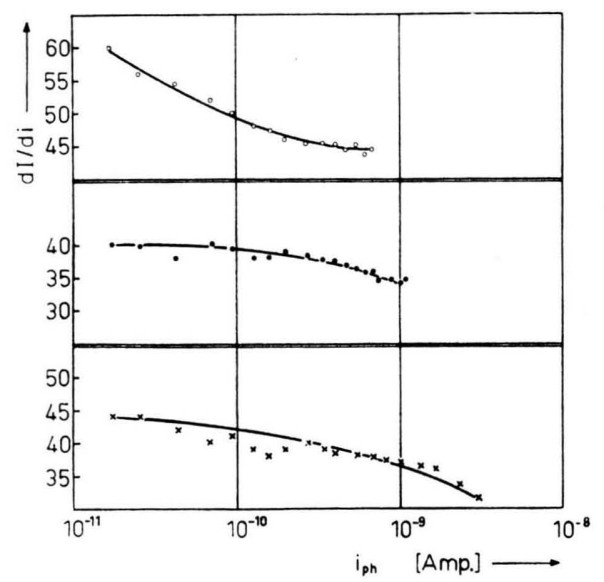

Abb. 5. Reziproke Steigung der Photostrom-Beleuchtungs-Charakteristisk $\mathrm{d} I / \mathrm{d} i$.

Die Messungen wurden mit weißem Licht einer Punktlichtlampe (Osram 55 V, 2 A) sowie mit den Quecksilberlinien 5460 und $4358 \AA$ durchgeführt. Ein Beispiel solcher Messungen ist in Tab. 4 und den Abb. 6 und 7 für den 10-stufigen Vervielfacher nach Dr. Schaetti, Betriebsspannung $2100 \mathrm{~V}$, Dunkelstrom $5 \cdot 10^{-8} \mathrm{~A}$ angegeben. Dieser Vervielfacher besitzt eine sehr große, auf das Glas aufge- 
dampfte Kathode. Da diese sehr dünn ist, war zu erwarten, daß eine Abhängigkeit der Charakteristik von der Bestrahlungsstelle beobachtet wurde.

a) Grüne Quecksilberlinie (5460 $\AA$ ),

b) Blaue Quecksilberlinie (4358 $\AA$ ),

c) Weißes Licht.

Diese Annahme erwies sich als richtig. Bei Einstrahlung unmittelbar an der Kathodenzuführung zeigte der Vervielfacher strenge Proportionalität innerhalb der Meßgenauigkeit, im andern Fall waren die Abweichungen, besonders bei Benutzung von weißem Licht, außerordentlich groß. Der Vervielfacher nach Dr. Maurer erwies sich als sehr gut linear, von den vier RCA-Vervielfachern war nur

a) Grüne Quecksilberlinie (5460 $)$

\begin{tabular}{|c|c|c|c|c|}
\hline $\begin{array}{c}i_{\mathrm{Ph}} \\
\text { (Ampere) }\end{array}$ & $\boldsymbol{I}_{\mathrm{gem} .}$ & $I_{\text {ber. }}$ & $\mathrm{d} \boldsymbol{I} / \mathrm{d} \boldsymbol{i}$ & $\frac{I_{\text {gem. }}-I_{\text {ber. }}}{I_{\text {gem. }}} \cdot 100$ \\
\hline $4,00 \cdot 10^{-6}$ & 1368,0 & 1368,0 & 35,7 & - \\
\hline 3,00 & $\begin{array}{l}1000,0 \\
1011,0\end{array}$ & $\begin{array}{l}100 \%, 0 \\
1022,0\end{array}$ & $\begin{array}{l}35,0 \\
35,0\end{array}$ & $-1,1$ \\
\hline 2,50 & 836,2 & 849,0 & 34,7 & $-1,6$ \\
\hline 2,00 & 662,7 & 676,0 & 34,5 & $-2,1$ \\
\hline 1,50 & 490,2 & 503,0 & 34,0 & $-2,6$ \\
\hline 1,25 & 404,5 & 416,5 & 34,0 & $-3,0$ \\
\hline $1,00 \cdot 10^{-6}$ & 319,4 & $\begin{array}{l}710,0 \\
330,0\end{array}$ & $\begin{array}{l}5+, 0 \\
34,0\end{array}$ & $\begin{array}{r}r, 0 \\
-3,2\end{array}$ \\
\hline $8,00 \cdot 10^{-7}$ & 251,4 & 260,8 & $\begin{array}{l}33,8 \\
33,8\end{array}$ & $-3,6$ \\
\hline 6,0 & 183,7 & 191,6 & 33,6 & $-4,1$ \\
\hline 5,0 & 150,1 & 157,0 & 33,5 & $-4,8$ \\
\hline 4,0 & 116,6 & 122,0 & 33,4 & $-4,4$ \\
\hline 3,0 & 83,15 & 87,00 & 33,4 & $-4,4$ \\
\hline 2,5 & 66,52 & 69,50 & $\begin{array}{l}33,4 \\
33,3\end{array}$ & $-4,3$ \\
\hline 2,0 & 49,85 & 52,10 & $\begin{array}{l}33,2 \\
33,2\end{array}$ & $-4,4$ \\
\hline 1,5 & 33,22 & 34,78 & 33,0 & $-4,4$ \\
\hline 1,25 & 24,96 & 26,13 & 33,0 & $-4,4$ \\
\hline $1,0 \cdot 10^{-7}$ & 16,71 & 17,48 & 33,1 & $-4,4$ \\
\hline $9,0 \cdot 10^{-8}$ & 13,40 & 14,02 & 33,0 & $-4,4$ \\
\hline 8,0 & 10,10 & 10,51 & $\begin{array}{l}33 \\
33\end{array}$ & $\begin{array}{r}-4,1 \\
-4,1\end{array}$ \\
\hline 7,0 & 6,80 & 7,09 & 33 & $-4,1$ \\
\hline 6,5 & 5,15 & 5,36 & 33 & $-3,9$ \\
\hline 6,0 & 3,50 & 3,63 & 33 & $-3,6$ \\
\hline 5,5 & 1,85 & 1,90 & 33 & $-2,6$ \\
\hline 5,2 & 0,86 & 0,86 & & - \\
\hline
\end{tabular}

b) Blaue Quecksilberlinie ( $4358 \AA$ )

\begin{tabular}{|c|c|c|c|c|}
\hline$i_{\mathrm{Ph}}$ & $I_{\text {gem. }}$ & $I_{\text {her. }}$ & $\mathrm{d} \boldsymbol{I} / \mathrm{d} i$ & $\frac{I_{\text {gem. }}-I_{\text {ber. }}}{I_{\text {kem. }}} \cdot 100$ \\
\hline $1,50 \cdot 10^{-6}$ & 453,4 & 453,4 & 32,9 & - \\
\hline 1,25 & 371,1 & 375,3 & 32,4 & $-1,1$ \\
\hline $1,00 \cdot 10^{-6}$ & 290,2 & 297,2 & 31,4 & $-2,3$ \\
\hline $8,00 \cdot 10^{-7}$ & 227,4 & 234,7 & 30,7 & $-2,9$ \\
\hline 6,00 & 166,0 & 172,2 & 30,4 & $-3,3$ \\
\hline 4,00 & 105,2 & 109,7 & 29,9 & $-4,1$ \\
\hline 3,00 & 75,32 & 78,56 & 30,1 & $-4,0$ \\
\hline 2,50 & 60,26 & 62,84 & 30,1 & $-4,3$ \\
\hline 2,00 & 45,23 & 47,22 & 29,9 & $-4,1$ \\
\hline 1,50 & 30,27 & 31,60 & 29,9 & $-4,0$ \\
\hline 1,25 & 22,80 & 23,79 & 30,0 & $-4,2$ \\
\hline 1,10 & 18,30 & 19,11 & 29,8 & $-4,0$ \\
\hline $1,00 \cdot 10^{-7}$ & 15,32 & 15,99 & 29,8 & $\begin{array}{r}-4,0 \\
-4,0\end{array}$ \\
\hline $9,00 \cdot 10^{-8}$ & 12,34 & 12,86 & 29,8 & $\begin{array}{r}-4,2 \\
-4,2\end{array}$ \\
\hline 8,00 & 9,36 & 9,76 & 29,7 & $-4,1$ \\
\hline 7,50 & 7,87 & 8,20 & 29,8 & $-4,0$ \\
\hline 7,00 & 6,38 & 6,64 & 29,9 & $-4,0$ \\
\hline 6,50 & 4,89 & 5,07 & 30,0 & $\begin{array}{r}-3,5 \\
-3,5\end{array}$ \\
\hline 6,00 & 3,39 & 3,50 & 29,8 & $-3,1$ \\
\hline 5,50 & 1,90 & 1,94 & 29,6 & $-2,1$ \\
\hline 5,25 & 1,16 & 1,16 & - & - \\
\hline
\end{tabular}

c) Weißes Licht

\begin{tabular}{|c|c|c|c|c|}
\hline$i_{\mathrm{Ph}}$ & $I_{\text {gem. }}$ & $I_{\text {ber. }}$ & $\mathrm{d} I / \mathrm{d} i$ & $\frac{I_{\text {gem. }}}{I} \frac{-I_{\text {ber. }}}{I \text { em. }} \cdot 100$ \\
\hline $5,00 \cdot 10^{-5}$ & 44580 & 44580 & 107 & - \\
\hline 4,00 & 33850 & 35650 & 100 & $-4,7$ \\
\hline 3,00 & 23830 & 26720 & 94,6 & $-11,5$ \\
\hline 2,50 & 19100 & 22260 & 90,4 & $-14,0$ \\
\hline 2,00 & 14580 & 17800 & $\begin{array}{l}80,7 \\
85,2\end{array}$ & $\begin{array}{l}-18,0 \\
-18,0\end{array}$ \\
\hline 1,50 & 10320 & 13340 & 80,1 & $\begin{array}{l}-22,7 \\
\end{array}$ \\
\hline 1,25 & 8317 & 11110 & 76,5 & $-25,1$ \\
\hline $1,00 \cdot 10^{-5}$ & 6404 & 8880 & 73,2 & $-27,8$ \\
\hline $7,50 \cdot 10^{-6}$ & 4572 & 6648 & 67,7 & $-31,3$ \\
\hline 6,00 & 3546 & 5308 & 66,6 & $-33,0$ \\
\hline 5,00 & 2880 & 4415 & $\begin{array}{l}64,8 \\
64,8\end{array}$ & $\begin{array}{r}-34,8 \\
-34,0\end{array}$ \\
\hline 4,00 & 2232 & 3522 & $\begin{array}{l}61,2 \\
6\end{array}$ & $-36,7$ \\
\hline 3,00 & 1620 & 2629 & 59,2 & $-38,4$ \\
\hline 2,50 & 1324 & 2183 & 57,4 & $-39,4$ \\
\hline 2,00 & 1037 & 1737 & 56,2 & $-40,2$ \\
\hline 1,50 & 755,8 & 1285 & 55,0 & $-41,4$ \\
\hline $\begin{array}{l}1,25 \\
1,25\end{array}$ & $\begin{array}{l}100, \circ \\
618,2\end{array}$ & 1064 & $\begin{array}{l}54,0 \\
54,0\end{array}$ & 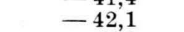 \\
\hline $1,00 \cdot 10^{-6}$ & 483,3 & 842,3 & 53,1 & $\begin{array}{r}-42,9 \\
-42\end{array}$ \\
\hline $9,00 \cdot 10^{-7}$ & 430,2 & 743,0 & 52,8 & $\begin{array}{l}72,0 \\
-42,3\end{array}$ \\
\hline 8,00 & 377,4 & 653,8 & 50,6 & $-42,4$ \\
\hline 7,50 & 352,1 & 609,2 & 52,4 & $-42,1$ \\
\hline 7,00 & 325,9 & 564,6 & 52,1 & $-42,5$ \\
\hline 6,00 & 273,8 & 475,3 & 52,1 & $-42,3$ \\
\hline 5,00 & 221,7 & 386,0 & 52,1 & $-43,0$ \\
\hline 4,00 & 169,6 & 296,7 & 51,6 & $\begin{array}{r}-42,7 \\
-\end{array}$ \\
\hline 3,00 & 118,0 & 207,4 & 51,2 & -429 \\
\hline 2,50 & 92,45 & 162,8 & 50,8 & $-43,2$ \\
\hline 2,00 & 67,04 & 118,2 & 50,8 & $-43,2$ \\
\hline 1,50 & 41,63 & 73,63 & 50,1 & $-43,4$ \\
\hline 1,25 & 29,10 & 51,31 & 50,2 & $-43,3$ \\
\hline $1,00 \cdot 10^{-7}$ & 16,55 & 28,99 & 50,1 & $-42,9$ \\
\hline $9,00 \cdot 10^{-8}$ & 11,54 & 20,07 & 50,0 & -42.5 \\
\hline 8,00 & 6,54 & 11,15 & 50,0 & $-41,2$ \\
\hline 6,85 & 0,79 & 0,79 & - & \\
\hline
\end{tabular}

Tab. 4. Bestrahlungsstelle im Abstand von $2 \mathrm{~cm}$ von der Kathodenzuführung.

einer (Nr. IV) linear, die andern zeigten Abweichungen bis zu $20 \%$, in einem Fall (Nr. VI bei weißem Licht) sogar bis zu 50\% Abweichungen von der Proportionalität. Der Intensitätsbereich der Beleuchtung erstreckte sich immer über etwa 4 Zehnerpotenzen, jedoch wurden die Vervielfacher niemals voll belastet.

Im folgenden seien die möglichen Ursachen für Abweichungen einer Zelle von der Proportionalitätsgeraden kurz zusammengestellt. Im allgemeinen wird nicht nur eine dieser Ursachen vorliegen, sondern mehrere werden zusammenwirken.

Unter Ermüdungserscheinungen versteht man eine Abnahme oder Zunahme der Empfindlichkeit der Zelle mit der Beobachtungszeit. Nach R o s en berg ${ }^{20}$ nimmt die Ermüdung mit steigender Feldstärke bei gleichbleibender Intensität und bei konstanter Feldstärke mit zunehmender Beleuchtungsstärke zu. Diese Ermüdung wird allgemein darauf zurückgeführt, daß die Kathode eine Gasschicht ad- oder absorbiert, in welcher eine starke Elektronenabsorption stattzufinden scheint. Es steht nicht fest, ob die positiven Gasionen mehr oder weniger tief in die Kathode eindringen (Absorption), oder ob sie nur 
an der Oberfläche haften (Adsorption), ob sie entladen werden oder ihre Ladung behalten und damit eine positiv geladene elektrische Doppelschicht über

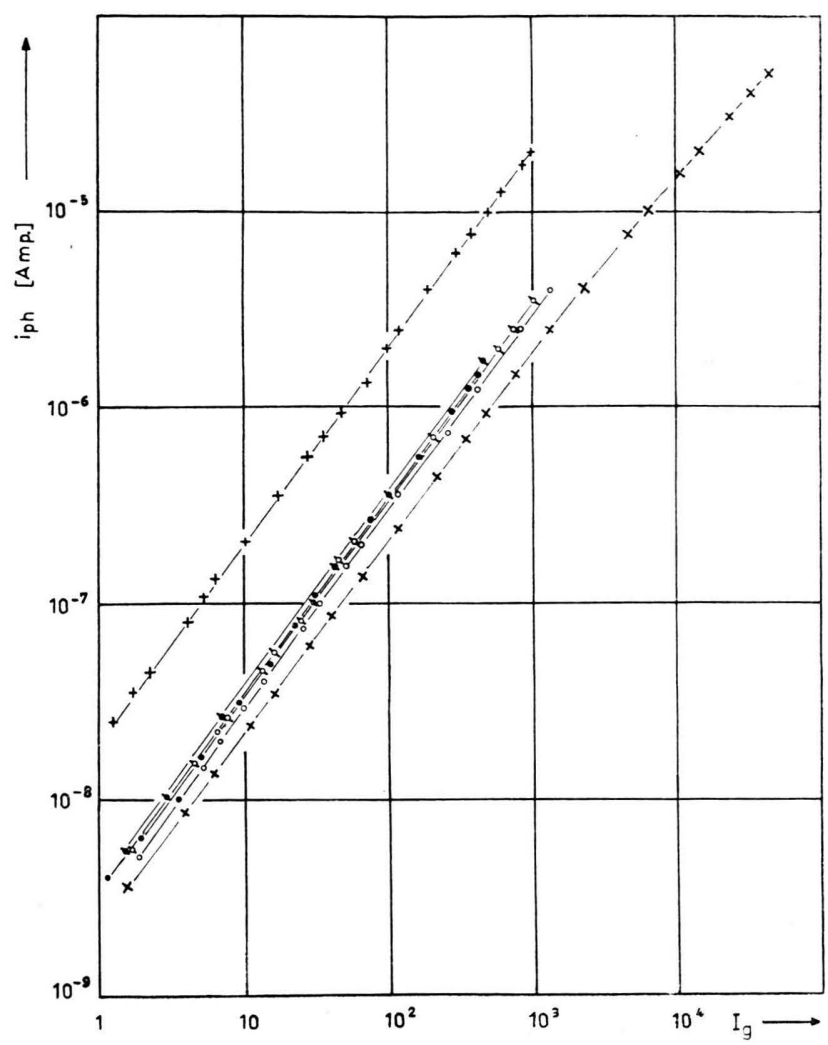

Abb. 6. Abhängigkeit des Photostroms von der Beleuchtungsstärke beim 10-stufigen Vervielfacher nach Dr. Schaetti.
a) $\bigcirc$ grüne $\mathrm{Hg}$-Linie
- blaue Hg-Linie
$x$ weißes Licht
b) - - - grüne Hg-Linie
-๑- blaue $\mathrm{Hg}$-Linie weißes Licht

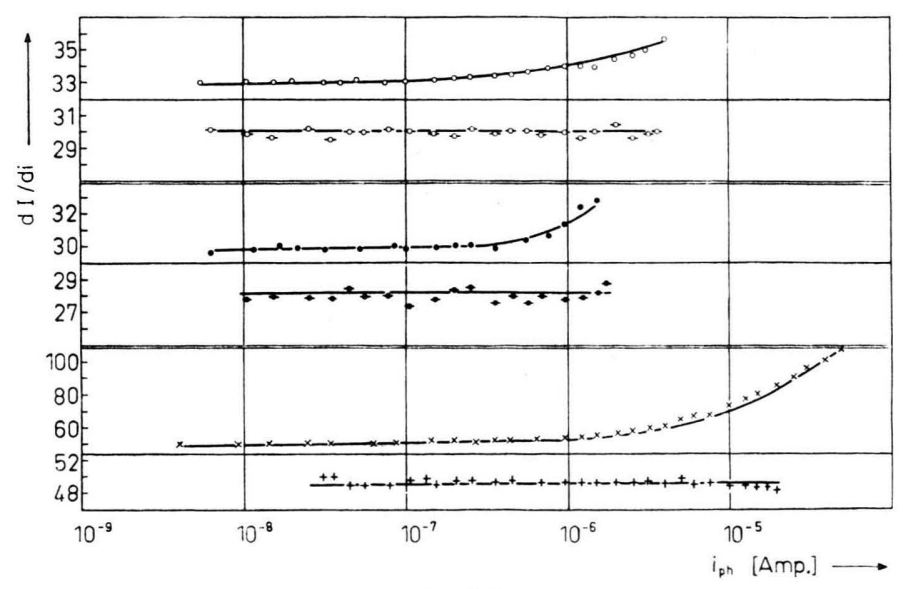

Abb. 7. Reziproke Steigung der Photostrom-Beleuchtungs-Charakteristik d $I / d i$ (Bezeichnung der Meßpunkte wie bei Abb. 6). der Kathode bilden. Wahrscheinlich finden beide Effekte gleichzeitig statt. Die Gasschicht wird sich durch Diffusion von der Oberfläche zu lösen versuchen. Solange weniger positive Ionen die Kathode verlassen als ankommen, steigt die Ermüdung an. Herrscht Gleichgewicht zwischen ankommenden und wegdiffundierenden Gasionen, so bleibt die Empfindlichkeit konstant. Die Wirkung dieser Ermüdung, die vor allem bei gasgefüllten Zellen auftritt, wird eine Abnahme der Empfindlichkeit mit steigender Beleuchtungsstärke sein. In einem gewissen, relativ engen Bereich wird Konstanz der Empfindlichkeit zu beobachten sein.

Bei Vervielfachern, und vor allem bei denen mit Cs-Sb-Schichten, tritt eine ganz andere Art von Ermüdung auf ${ }^{38}$. Die Cs-Sb-Schichten werden durch Einwirkung von Cs-Dampf auf mit Sb belegte Elektroden hergestellt. Das Maximum der Empfindlichkeit ist dann erreicht, wenn die Verbindung $\mathrm{SbCs}_{3}$ erreicht ist, die sich aber nur unterhalb von $220^{\circ} \mathrm{C}$ bildet. Bei Mangel oder Überschuß von Cs ist die Empfindlichkeit geringer. Treffen nun Elektronen auf eine mit Cs belegte Elektrode auf, so wird überschüssiges Cs teilweise verdampfen. Dadurch nähert sich die Zusammensetzung der Schicht mehr dem stöchiometrischen Verhältnis 3:1, d. h. die Empfindlichkeit steigt an, die „Proportionalitätsgerade ist nach oben gekrümmt. Besitzt die Elektrode schon von vornherein die optimale Zusammensetzung, so tritt bei Elektronenbeschuß eine Verminderung der Empfindlichkeit mit der Zeit auf, da die Verbindung aufgespalten wird.

Auch zusammengesetzte Kathoden mit dicken Zwischenschichten zeigen Ermüdung. Die Ursache wird hier in einem elektrolytischen Hineinwandern der beim Primärprozeß (Photoionisation) gebildeten und noch nicht neutralisierten Alkaliionen in das Innere der Schicht gesehen $^{39}$, wobei die Oberfläche an ionisierbaren Atomen verarmt und damit die Empfindlichkeit absinkt. Für diese Anschauung spricht auch der Befund von Preston und Mc Dermott ${ }^{29}$, daß eine Vakuumzelle, die kein überschüssiges Alkalimetall enthält, auch nach Wochen und Monaten dieselbe Charakteristik zeigt.

${ }^{38}$ F. H. Marshall, J. W. Coltman u. L. P. Hunter, Rev. Sci. Instruments 18, 504 [1947].

${ }_{39}$ J. H. de Boer u. M. C. Teves, Z. Physik 73, 192 [1932]. 
Die Anode einer Photozelle besitzt im allgemeinen eine sehr kleine Fläche, und daher spielen in ihrer Umgebung Raumladungen eine sehr wesentliche Rolle. Die Raumladungskonzentration kann so groß werden, daß es unmöglich wird, Sättigung zu erreichen. Allerdings nimmt bei einer gewöhnlichen Photozelle die Empfindlichkeit um so langsamer $a b$, je höher die Beleuchtungsstärke ist, während die Raumladungen immer bedeutender werden. Andererseits beobachtet man bei nicht zu großen Zellspannungen bei Erhöhung der Spannung eine Verbesserung der Charakteristik, was durch Raumladungen zwanglos erklärt werden kann. Eine sehr wichtige Rolle spielen Raumladungen bei Vervielfachern. In den letzten Stufen des Vervielfachers wird die Stromstärke so groß, daß durch die Raumladung die elektronenoptische Abbildung der Elektroden aufeinander verloren geht, $d$. h. ein Teil der Elektronen an der folgenden Sekundärelektrode vorbeifliegen und damit aus dem Vervielfachungsproze $ß$ ausscheiden.

Der Einfluß der Temperatur auf die Empfindlichkeit einer Zelle ist im allgemeinen zu vernachlässigen. Lediglich in den Fällen, in denen die Wärmeableitung von der bestrahlten Schicht sehr schlecht ist, dürfte eine merkliche Temperaturänderung und damit Empfindlichkeitsänderung eintreten.

Infolge des hohen Dampfdrucks der Alkalimetalle kann sich auf den Wänden der Zelle eine dünne Alkalischicht ausbilden, die unter Umständen einen sehr hohen Widerstand besitzt. Diese Schicht ruft einmal eine Erhöhung des Dunkelstromes hervor, übt aber vor allem durch ihre Aufladung einen steuernden Einfluß auf den Photostrom aus. Steht der Film mit der Kathode in leitender Verbindung, so kann er zum Anlaß für Abweichungen der Zelle von der Proportionalität werden. Die Filme können einmal auf der Glaswand niedergeschlagen sein und damit einen hohen elektrischen Widerstand besitzen, oder sich auf der Kathode befinden und von ihr durch eine Halbleiterschicht getrennt sein. Dieser letzte Fall dürfte häufig bei Schichtkathoden eintreten, bei denen das Grundmetall vor dem Anbringen der Alkalischicht oxydiert wird, wobei sich hier und da dickere Oxydschichten nicht vermeiden lassen. Man kann annehmen, daß ein Teil des Photostroms von den normalen Teilen der Kathode kommt und damit (falls nicht noch andere Einflüsse vorliegen) dem Proportionalitätsgesetz gehorcht, der andere Teil von diesen Filmen. Bei Durchgang des Photostroms wird in ihnen ein Potentialgefälle entstehen, das so groß werden kann, daß ein Teil des Filmes keine Elektronen mehr zu emittieren vermag. Die Größe dieses Teiles variiert mit dem Photostrom. Dazu kommt noch die Ablenkung eines Teiles der Photoelektronen auf die positiven Teile des Films. Die Folge ist Nichtproportionalität. Preston und McDermott ${ }^{29}$ fanden für die Abhängigkeit der Empfindlichkeit $S$ von der Beleuchtungsstärke $I$ unter der Annahme derartiger dünner Filme eine Beziehung der Form $S=K_{1}+K_{2} /(I)^{1 / 2}$, wo $K_{1}$ und $K_{2}$ Konstanten sind. Mit steigender Intensität wird der Ausdruck $K_{2} /(I)^{1 / 2}$ klein gegen $K_{1}$, d. h. die Empfindlichkeit ändert sich bei hohen Intensitäten langsamer mit der Beleuchtungsstärke, ein Ergebnis, das in vielen Fällen experimentell bestätigt wurde.

Alle diese Einflüsse können zusammenwirken und dadurch eine Charakteristik hervorrufen, wie sie bei unseren Untersuchungen gefunden wurde.

Wie sich aus den Messungen ergibt, ist es zwar heute möglich, Vakuumzellen und -Vervielfacher, in selteneren Fällen auch gasgefüllte Zellen zu erhalten, die genügende Annäherung an die exakte Proportionalität zeigen, aber für den praktischen Gebrauch einer Photozelle, sei es für photometrische Zwecke großer Genauigkeit oder für frequenzgetreue Umsetzung von Lichtschwankungen in Stromschwankungen, ergibt sich trotzdem die Notwendigkeit der systematischen Prüfung jeder einzelnen Zelle unter den verschiedensten Bedingungen.

Der Deutschen Forschungsgemeinschaft haben wir für Bereitstellung von Apparaten zu danken. 